\title{
ESTIMATIVA DA PRODUTIVIDADE DA CANA-DE-AÇÚCAR UTILIZANDO O SEBAL E IMAGENS LANDSAT
}

\author{
RICARDO GUIMARÃES ANDRADE ${ }^{1}$, GILBERTO SEDIYAMA $^{2}$, VICENTE PAULO SOARES ${ }^{3}$, JOSÉ \\ MARINALDO GLERIANI ${ }^{3}$, SADY JUNIOR MARTINS DA COSTA MENEZES ${ }^{4}$
}

${ }^{1}$ Empresa Brasileira de Pesquisa Agropecuária (EMBRAPA), Campinas, São Paulo, Brasil

${ }^{2}$ Universidade Federal de Viçosa (UFV), Departamento de Engenharia Agrícola, Viçosa, Minas Gerais, Brasil

${ }^{3}$ UFV, Departamento de Engenharia Florestal, Viçosa, Minas Gerais, Brasil

${ }^{4}$ Universidade Federal Rural do Rio de Janeiro (UFRJ), Instituto de Três Rios, Três Rios, Rio de Janeiro, Brasil

ricardo.andrade@embrapa.br, g.sediyama@ufv.br,vicente@ufv.br, gleriani@ufv.br, sadymenezes@yahoo.com.br

Recebido Agosto de 2013 - Aceito Novembro de 2013

\begin{abstract}
RESUMO
As técnicas de sensoriamento remoto têm sido muito promissoras para o desenvolvimento de medidas mais confiáveis e economicamente viáveis da produção vegetal em larga escala. O algoritmo SEBAL (Surface Energy Balance Algorithm for Land) tem como vantagem a obtenção de parâmetros biofísicos usando imagens de satélite e poucos dados observacionais. Este trabalho objetivou estimar a produtividade da cana-de-açúcar por meio da aplicação do algoritmo SEBAL e de imagens Landsat 5 TM. O estudo foi realizado em plantios de cana-de-açúcar da fazenda Boa Fé, localizada no Triângulo Mineiro, município de Conquista, Minas Gerais. A metodologia utilizada apresentou variação de desempenho nas estimativas de produtividade da cana-de-açúcar de cada gleba, provavelmente em decorrência de influências da dimensão dos talhões e da resolução espacial da imagem, e de variedades e épocas de plantio e colheita da cultura. No entanto, os resultados apontam que a metodologia tem potencial para ser aplicada em áreas extensas com limitada disponibilidade de dados meteorológicos. Palavras-chave: Cana-de-açúcar, Biomassa Vegetal, SEBAL, Sensoriamento Remoto.
\end{abstract}

\begin{abstract}
SUGARCANE YIELD ESTIMATES USING SEBAL AND LANDSAT IMAGES Remote sensing techniques have shown very promising results in the development of more trustworthy and economically viable large-scale agricultural production measurements. The Surface Energy Balance Algorithm for Land (SEBAL) has the advantage of obtaining biophysical parameters using satellite images and few observational data. This work aimed to estimate sugarcane production using the SEBAL algorithm and Landsat $5 \mathrm{TM}$ images. It was performed on sugarcane crops at the Boa Fé farm, located in Conquista, MG, Brazil. The used method showed varying performance in the sugarcane production estimates for each plot, probably due to the influences of the size of the plots and the spatial resolution of the image, and of varieties and crop planting and harvest dates. However, the results indicate the method has potential for application on large areas on which there is limited availability of meteorological data.
\end{abstract}

Keywords: Sugarcane, Biomass, SEBAL, Remote Sensing. 


\section{INTRODUÇÃO}

A cana-de-açúcar tem participação relevante na economia brasileira desde o período colonial, e o cenário atual aponta perspectivas de consolidação e expansão da utilização da cana-de-açúcar como fonte de matéria-prima na produção de energia renovável (Mello e Esperancini, 2012). Diante disso, a retomada de políticas agroenergéticas no País vem influenciando a redistribuição espacial da produção canavieira, com tendência de expansão em estados como Goiás, Mato Grosso, Mato Grosso do Sul e Minas Gerais (Carvalho e Marin, 2011). Essa crescente expansão tem contribuído para que o país se destaque como o maior produtor mundial de cana-de-açúcar (Vicente et al., 2012).

Para Guimarães et al. (2010), o Brasil tem forte potencial para ser o principal polo mundial de produção de biocombustíveis gerados a partir óleos vegetais e cana-deaçúcar. As características do território brasileiro (dimensão territorial, relevo, tipo de solo, clima, etc.) têm favorecido os investimentos no setor sucroalcooleiro, principalmente como forma de aproveitamento de áreas de pastagens degradadas.

Diante da crescente demanda dos mercados interno e externo pela produção de biocombustíveis e considerando-se a expansão da produção de cana-de-açúcar em diversas regiões do País, torna-se fundamental obter informações relacionadas à produção dessa commodity para auxiliar na gestão estratégica, tanto na escala local, quanto regional. A estimativa da produção agrícola pode ser de interesse relevante, por exemplo, na tomada de decisão das empresas quanto à logística de colheita, armazenamento e escoamento da produção.

Nas últimas décadas, a utilização de técnicas de sensoriamento remoto juntamente com modelos e algoritmos tem sido promissora para a obtenção de parâmetros biofísicos de superfície e predição da produção das culturas. $\mathrm{O}$ algoritmo SEBAL (Surface Energy Balance Algorithm for Land), desenvolvido por Bastiaanssen et al. (1998a, 1998b), tem sido aplicado em diversas pesquisas no Brasil, como na estimativa da água consumida em perímetro irrigado do Semiárido brasileiro (Silva et al., 2012), na avaliação de parâmetros biofísicos do Pantanal (Andrade et al., 2012) e em estudos relacionados ao balanço de energia e da evapotranspiração em plantios de eucalipto (Menezes et al., 2011), porém também é promissor em aplicações relacionadas com a estimativa da produtividade agrícola.

Bastiaanssen e Ali (2003) acoplaram no SEBAL o modelo de acúmulo de biomassa proposto por Monteith (1972), juntamente com o modelo de eficiência do uso da radiação estruturado por Field et al. (1995), com o objetivo de estimar a biomassa acima do solo na bacia Indus no Paquistão; o mesmo foi realizado por Samarasinghe (2003) no Sri Lanka.

Neste contexto, este trabalho objetivou estimar a produtividade da cana-de-açúcar por meio da aplicação de técnicas de sensoriamento remoto juntamente com o algoritmo SEBAL.

\section{MATERIAL E MÉTODOS}

A área de estudo está localizada na Fazenda Boa Fé, município de Conquista, MG (Figura 1). A fazenda tem área de aproximadamente 1.700 ha, e a maior parte é cultivada com cana-de-açúcar. A vegetação natural faz parte do Bioma Cerrado e os solos são classificados como Latossolo Vermelho, Latossolo Vermelho-Amarelo e Argissolo Vermelho-Amarelo.

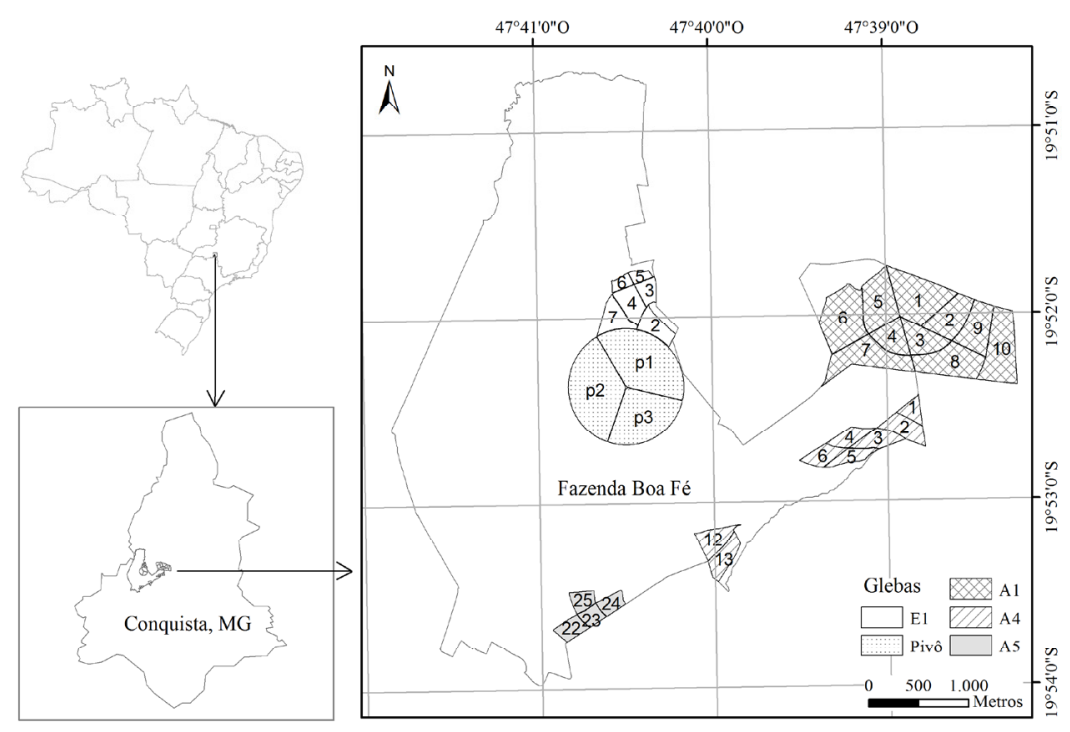

Figura 1 - Localização das glebas de plantios da cana-de-açúcar em talhões numerados em área da Fazenda Boa Fé, município de Conquista, MG. 
Na Figura 1 visualizam-se as glebas de plantio da canade-açúcar juntamente com a numeração de cada talhão. Já na Tabela 1 são apresentados os dados referentes a datas de plantio e colheita, variedades, números de talhões e cortes das glebas A1, A4, A5, E1 e Pivô.

Segundo a classificação internacional de Köppen, na região predomina o clima do tipo $\mathrm{Aw}$, ou seja, tropical quente e úmido com inverno frio e seco. A média anual da temperatura do ar é de $21,9^{\circ} \mathrm{C}$, e a média anual das temperaturas mínima e máxima do ar é de $13,2{ }^{\circ} \mathrm{C}$ e $29,1{ }^{\circ} \mathrm{C}$, respectivamente. Geralmente os meses de janeiro, fevereiro e dezembro são os mais chuvosos e os meses de junho, julho e agosto são os mais secos.

Para obtenção dos componentes do balanço de energia por meio do algoritmo SEBAL, foram utilizados dados meteorológicos (temperatura do ar, radiação e velocidade do vento) da Plataforma de Coleta de Dados (PCD) localizada no município de Miguelópolis, SP, pertencente à rede de estações meteorológicas do Centro de Previsão do Tempo e Estudos Climáticos (CPTEC).

Neste estudo, foram utilizadas imagens Landsat $5 \mathrm{TM}$ dos anos de 2004 a 2007 que não tinham cobertura de nuvens (Tabela 2). Essas imagens são compostas por sete bandas espectrais, têm resolução espacial de $30 \mathrm{~m}$ nas bandas 1, 2, $3,4,5$ e 7 e de $120 \mathrm{~m}$ na banda 6 (termal), e foram obtidas do catálogo de imagens do Instituto Nacional de Pesquisas Espaciais (INPE).

Após a obtenção das imagens, teve início uma série de passos relacionados à aplicação do SEBAL e demais modelos para estimativa da biomassa vegetal e, por conseguinte, obtenção da produtividade da cana-de-açúcar.

A aplicação do algoritmo SEBAL consiste em métodos baseados fisicamente no esquema de transferência da camada única para o calor sensível $(\mathrm{H})$ e em uma estimativa empírica para o fluxo de calor no solo $(\mathrm{G})$. O saldo de radiação $(\mathrm{Rn})$ é calculado por meio da reflectância e emitância da radiação, variável espacialmente. $\mathrm{O}$ fechamento do balanço de energia pixel a pixel é processado considerando-se o fluxo de calor latente (LE) como um resíduo da equação do balanço de energia, dado por:

$$
L E=R n-H-G
$$

em que LE, Rn, H e G são dados em $\mathrm{W} \mathrm{m}^{-2}$.

O saldo de radiação $(\mathrm{Rn})$ foi a primeira variável da equação do balanço de energia a ser obtida (Figura 2). Para tanto, aplicou-se a equação sugerida por Allen et al. (2002):

$$
R n=R_{s \downarrow}-\alpha \times R_{s \downarrow}+R_{L \downarrow}-R_{L \uparrow}-\left(1-\varepsilon_{o}\right) \times R_{L \downarrow}
$$

em que $R_{S \downarrow}$ é a radiação de onda curta incidente $\left(\mathrm{W} \mathrm{m}^{-2}\right), R_{L \downarrow}$ é a radiação de onda longa emitida pela atmosfera na direção da

\begin{tabular}{|c|c|c|c|c|c|}
\hline Gleba & $\mathrm{N}^{\mathrm{o}}$ do talhão & Variedades & $\begin{array}{l}\mathrm{N}^{0} \mathrm{de} \\
\text { cortes }\end{array}$ & Plantio & Colheita \\
\hline \multirow{2}{*}{ A1 } & \multirow{2}{*}{01 a 10} & \multirow{2}{*}{ RB 867515} & \multirow{2}{*}{1} & \multirow{2}{*}{$25 / 10 / 2006$} & $31 / 8 / 2007$ \\
\hline & & & & & $27 / 9 / 2007$ \\
\hline \multirow{4}{*}{ A4 } & 01 a 04 & RB 86-7515 & 2 & 9 a12/3/2005 & $1 / 5 / 2007$ \\
\hline & 05 e 06 & SP $81-3250$ & 2 & 14 a $16 / 3 / 2005$ & $16 / 6 / 2007$ \\
\hline & 12 & RB 92-8064 & 2 & 14 a $16 / 3 / 2005$ & $31 / 5 / 2007$ \\
\hline & 13 & $\begin{array}{c}\text { IAC 86-2480 e } \\
\text { RB 92-8064 }\end{array}$ & 2 & 14 a $16 / 3 / 2005$ & $24 / 6 / 2007$ \\
\hline \multirow{2}{*}{ A5 } & 22,22 e 25 & \multirow{2}{*}{ RB 92-579 } & \multirow{2}{*}{1} & \multirow{2}{*}{16 a $17 / 4 / 2006$} & 25 a 30/5/2007 \\
\hline & 24 & & & & $24 / 6 / 2007$ \\
\hline \multirow{3}{*}{ E1 } & $\begin{array}{c}01 \\
02 \text { a } 04\end{array}$ & RB 867515 & 3 & $23 / 3 / 04$ & $\begin{array}{l}26 / 6 / 2007 \\
11 / 6 / 2007\end{array}$ \\
\hline & 05 e 06 & \multirow{2}{*}{ RB 867515} & 2 & $15 / 3 / 05$ & $26 / 6 / 2007$ \\
\hline & 07 & & 3 & $23 / 3 / 04$ & $11 / 6 / 2007$ \\
\hline Pivô & $\mathrm{p} 1, \mathrm{p} 2$ e p3 & RB 867515 & 1 & $10 / 8 / 2006$ & 11 a $18 / 8 / 2007$ \\
\hline
\end{tabular}

Tabela 1 - Dados do número do talhão, variedade, número de cortes e datas de plantio e colheita nas glebas A1, A4, A5, E1 e Pivô

Tabela 2 - Datas das imagens Landsat 5 TM de órbita 220 e ponto 74 que foram obtidas do catálogo de imagens do INPE.

\begin{tabular}{ccccc}
\hline Ano & 2004 & 2005 & 2006 & 2007 \\
\hline \multirow{2}{*}{ Dia/Mês } & 14 e & $22 / 2,11 / 4$, & $24 / 1,14$ e $30 / 4,16 / 5,1$ e $17 / 6,3$ e & $14 / 4,3 / 5,4$ e 20/6, \\
& $30 / 8$ & $14 / 6,1 / 8$ & $19 / 7,4 / 8,5 / 9$ & $6 / 7,7 / 8$ \\
\hline
\end{tabular}


superfície $\left(\mathrm{W} \mathrm{m}^{-2}\right), R_{L \uparrow}$ é a radiação de onda longa emitida na direção da atmosfera $\left(\mathrm{W} \mathrm{m}^{-2}\right), \varepsilon_{\mathrm{o}}$ é a emissividade da superfície (adimensional) e $\alpha$ é o albedo da superfície (adimensional). Allen et al. (2002) apresentam em detalhes os procedimentos envolvidos na obtenção do saldo de radiação ( $\mathrm{Rn})$.

Após o cálculo de $\mathrm{Rn}$, procedeu-se com a aplicação da equação empírica sugerida por Bastiaanssen (2000) para estimativa do fluxo de calor no solo $\left(\mathrm{G}\right.$, em $\left.\mathrm{W} \mathrm{m}^{-2}\right)$, dada por:

$$
\mathrm{G}=\left[\frac{\mathrm{T}_{\mathrm{s}}}{\alpha} \times\left(0,0038 \times \alpha+0,0074 \times \alpha^{2}\right)\left(1-0,98 \times \mathrm{NDVI}^{4}\right)\right] \times \mathrm{Rn}
$$

em que $\mathrm{T}_{\mathrm{S}}$ é a temperatura da superfície $\left({ }^{\circ} \mathrm{C}\right), \alpha$ é o albedo da superfície (adimensional), NDVI é o índice de vegetação da diferença normalizada e Rn é o saldo de radiação. Para efeito de correção dos valores do fluxo de calor no solo para corpos d'água (NDVI $<0$ ), considerou-se $\mathrm{G}=0,3 \mathrm{Rn}$ (Silva e Bezerra, 2006). Obtido o valor de $\mathrm{G}$, iniciou-se uma nova série de passos necessários para a obtenção do fluxo de calor sensível $(\mathrm{H})$.

Para estimativa de $\mathrm{H}$, o processo teve início considerando-se a condição de atmosfera neutra. Primeiramente, foram aplicadas as expressões sugeridas por Allen et al. (2002) para obter o parâmetro de rugosidade inicial ( $\mathrm{z}_{\text {om }}$ inicial), a velocidade de fricção inicial ( $u^{*}$ inicial), a velocidade do vento a uma altura (z) de $100 \mathrm{~m}$, chamada de blending height (altura de mistura, onde se assume que os efeitos da rugosidade da superfície são desprezíveis), e a resistência aerodinâmica inicial ( $r_{a h}$ inicial). Nos passos seguintes, foi utilizado o parâmetro de rugosidade $\left(\mathrm{z}_{\mathrm{om}}\right)$ obtido em função do SAVI (Soil Adjusted Vegetation Index).

O SEBAL utiliza dois pixels denominados "pixels âncoras" para fixar condições limite para o balanço de energia. Esses pixels são chamados de "quente" e "frio" e ficam situados na área de estudo. O pixel "frio" pode ser selecionado em uma superfície de cultura bem irrigada que cobre completamente o solo com a vegetação ou na superfície de um lago. Neste caso, selecionou-se o pixel "frio" sobre a superfície de um lago. A temperatura do ar próxima à superfície e a temperatura da superfície são consideradas iguais para esse pixel. Assim, assumiu-se valor zero para o fluxo de calor sensível $(\mathrm{H})$ e determinou-se o fluxo de calor latente máximo por meio da equação:

$$
L E_{\text {frio }}=R n-G
$$

O pixel "quente" foi selecionado em um campo agrícola seco, com solo exposto ou sem vegetação, onde considerou-se valor zero para o fluxo de calor latente (LE) e, assim, obteve-se o fluxo de calor sensível máximo ao empregar a equação:

$$
H_{\text {quente }}=R n-G=\frac{\rho \times c_{p} \times\left(a+b \times T_{s}\right)}{r_{a h}}
$$

em que a e b são parâmetros de ajuste, $\rho$ é a densidade do ar úmido $\left(\mathrm{kg} \mathrm{m}^{-3}\right), \mathrm{c}_{\mathrm{p}}$ é o calor específico do ar à pressão constante (1.004 $\left.\mathrm{J} \mathrm{kg}^{-1} \mathrm{~K}^{-1}\right), \mathrm{r}_{\mathrm{ah}}$ é a resistência aerodinâmica, $\mathrm{T}_{\mathrm{s}}$ é a temperatura da superfície em Kelvin (K).

Por meio dos pixels âncoras foi possível encontrar os coeficientes de correlação a e b para obtenção de dT em cada pixel da imagem. Como no pixel frio $\mathrm{dT}=0$, ou seja, $\mathrm{a}+\mathrm{b} \mathrm{T}_{\mathrm{s}}=0$, tem-se um sistema com duas equações e duas incógnitas, o que possibilitou o cálculo de a e b. Logo, obteve-se o fluxo de calor sensível inicial $\left(\mathrm{H}_{\text {inicial }}\right)$ :

$$
H_{\text {inicial }}=\rho \times c_{p} \times \frac{d T}{r_{a h}}
$$

A próxima etapa foi considerar a condição de estabilidade atmosférica, realizando correções nos valores de $\mathrm{H}$ em processo iterativo. Para tanto, foi aplicada a teoria da similaridade de Monin-Obukhov (L, em m) para saber qual é a condição de

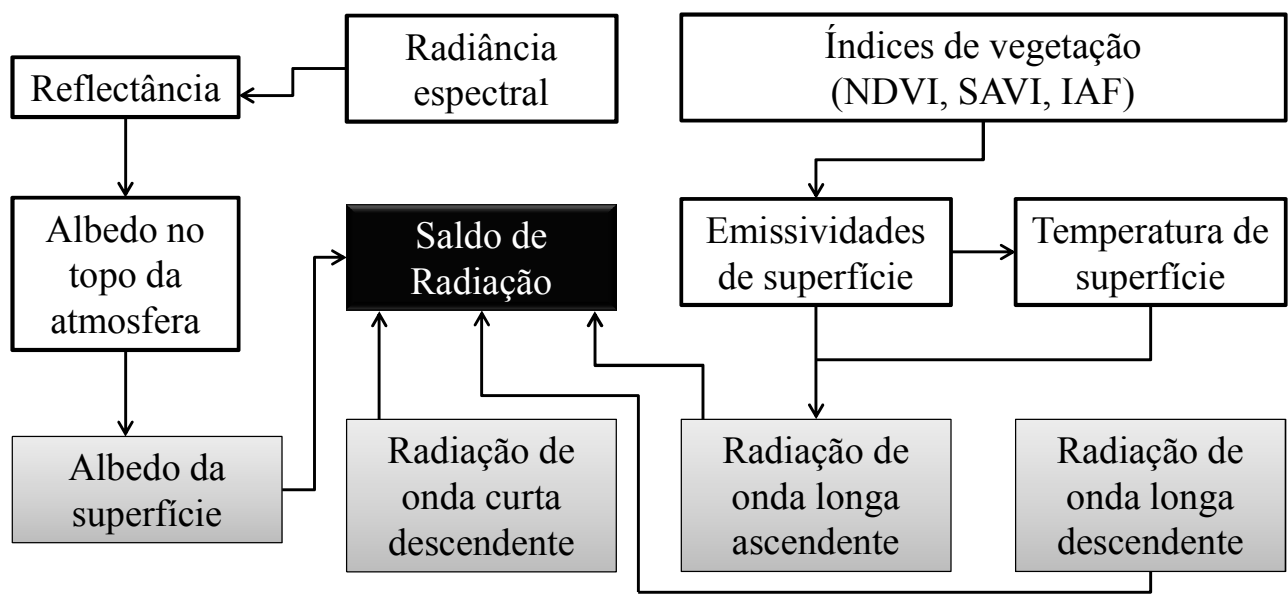

Figura 2 - Fluxograma para estimativa do saldo de radiação de superfície. 
estabilidade da atmosfera, ou seja, se ela é instável $(\mathrm{L}<0)$, estável $(\mathrm{L}>0)$ ou neutra $(\mathrm{L}=0)$, conforme expressão:

$$
\mathrm{L}=-\frac{\rho \times \mathrm{c}_{\mathrm{p}} \times \mathrm{u}_{*}{ }^{3} \times \mathrm{T}_{\mathrm{s}}}{\mathrm{k} \times \mathrm{g} \times \mathrm{H}}
$$

em que g é a aceleração da gravidade $\left(9,81 \mathrm{~m} \mathrm{~s}^{-2}\right)$ e $\mathrm{k}$ é a constante de von Karman $(0,41), \rho$ é a densidade do ar úmido $\left(\mathrm{kg} \mathrm{m}^{-3}\right), \mathrm{c}_{\mathrm{p}}$ é o calor específico do ar à pressão constante (1.004 $\left.\mathrm{J} \mathrm{kg}^{-1} \mathrm{~K}^{-1}\right), \mathrm{T}_{\mathrm{s}}$ é a temperatura da superfície em kelvin $(\mathrm{K}), \mathrm{u}^{*}$ é a velocidade de fricção e H é o fluxo de calor sensível.

Em seguida, por meio das formulações sugeridas por Allen et al. (2002), foi possível obter os valores das correções de estabilidade para o transporte de momentum $\left(\psi_{\mathrm{m}}\right)$ e de calor sensível $\left(\psi_{\mathrm{h}}\right)$. Posteriormente, estimou-se a velocidade de fricção $\left(u^{*}\right)$ considerando a condição atmosférica:

$$
\mathrm{u}_{*}=\frac{\mathrm{k} \times u_{100}}{\ln \left(\frac{z}{\mathrm{z}_{0 \mathrm{~m}}}\right)-\psi_{\mathrm{m}(100 \mathrm{~m})}}
$$

De posse do valor corrigido de $\mathrm{u}^{*}$, obteve-se o valor corrigido de $r_{a h}$ :

$$
\mathrm{r}_{\mathrm{ah}}=\frac{\ln \frac{\mathrm{z}_{2}}{\mathrm{z}_{1}}-\psi_{\mathrm{h}(2 \mathrm{~m})}+\psi_{\mathrm{h}(0,1 \mathrm{~m})}}{\mathrm{u}_{*} \mathrm{k}}
$$

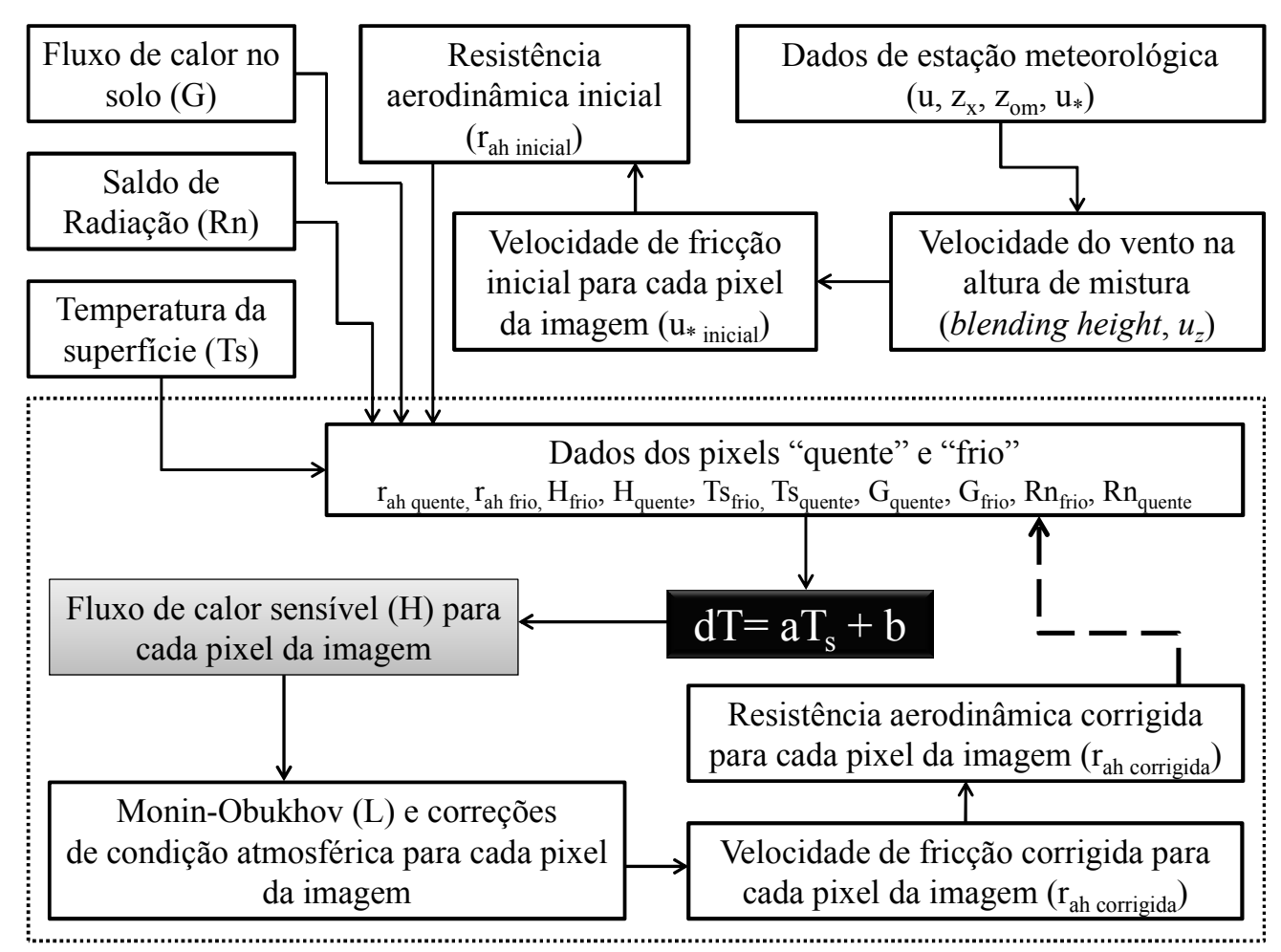

Figura 3 - Fluxograma do processo iterativo para estimativa do fluxo de calor sensível $(H)$.
Em seguida, retornou-se ao cálculo da função da diferença de temperatura (dT), repetindo-se os procedimentos mencionados anteriormente até que se observasse estabilidade nos valores sucessivos de $d T$ e $r_{\text {ah }}$ para o pixel quente. Por fim, obteve-se o fluxo de calor latente (LE) como um resíduo da equação clássica do balanço de energia (Equação 1). Na Figura 3 , tem-se o fluxograma dos passos necessários para a estimativa do fluxo de calor sensível $(\mathrm{H})$ por meio de processo iterativo.

Com as componentes do balanço de energia, estimou-se a fração evaporativa $(\lambda)$, dada por:

$$
\lambda=W=\frac{L E}{L E+H}=\frac{L E}{R n-G}
$$

A fração evaporativa foi utilizada para a estimativa da biomassa vegetal. Para tanto, realizou-se a estimativa da radiação fotossinteticamente ativa (PAR, em $\mathrm{W} \mathrm{m}^{-2}$ ) por meio da equação (Bastiaanssen e Ali, 2003):

$$
P A R=0,48 \times K_{\text {diário }}^{\downarrow}
$$

a qual expressa a PAR como $48 \%$ da radiação de onda curta incidente em um período de 24 horas $\left(K_{\text {dírio }}^{\downarrow}\right)$. Sabe-se que a radiação absorvida e utilizada pelos pigmentos da planta (radiação fotossinteticamente ativa absorvida, APAR) encontra-se na faixa correspondente ao espectro de luz visível $(0,4$ e $0,7 \mu m)$. Também é sabido que a radiação na faixa do infravermelho próximo é fortemente refletida pela vegetação. Em 
consequência dessa absorção seletiva e reflexão pelas plantas, a combinação da reflectância do visível e do infravermelho próximo deu origem aos chamados índices de vegetação, dos quais o NDVI é um dos mais utilizados na estimativa da fração da radiação PAR, interceptada (FPAR, em $\mathrm{W} \mathrm{m}^{-2}$ ) e absorvida (APAR, em $\mathrm{W} \mathrm{m}^{-2}$ ) pelo dossel das plantas. Com base nesse conceito, a determinação da FPAR foi realizada por meio da expressão empírica sugerida por Daughtry et al. (1992):

$$
F P A R=-0,161+1,257 \times N D V I
$$

Após as estimativas de PAR e FPAR, pode-se então determinar a APAR (em $\mathrm{W} \mathrm{m}^{-2}$ ) empregando-se a equação:

$$
A P A R=F P A R \times P A R
$$

Neste ponto, ao substituir o escalar da disponibilidade hídrica (W) pela fração evaporativa no modelo proposto por Field et al. (1995), foi possível calcular a eficiência do uso da radiação $\left(\varepsilon_{f}\right)$ pela expressão:

$$
\varepsilon_{f}=\varepsilon_{f}^{*} \times T_{1} \times T_{2} \times \lambda
$$

em que $\varepsilon_{f}^{*}$ é o valor médio da máxima eficiência do uso da radiação, igual a 2,5 $\mathrm{g} \mathrm{MJ}^{-1}$ (Bastiaanssen e Ali, 2003; Teixeira et al., 2012); $\lambda$ é a fração evaporativa (adimensional); e $\mathrm{T}_{1} \mathrm{e}$ $\mathrm{T}_{2}$ são escalares de temperatura obtidos por meio da aplicação das expressões:

$$
\begin{aligned}
T_{1}= & 0,8+0,02 \times T_{\text {otima }}-0,0005 \times T_{\text {otima }}^{2} \\
T_{2}= & \frac{1}{1+\exp \left\{0,2 \times\left(T_{\text {otima }}-10-T_{\text {mensal }}\right)\right\}}: \\
& \times \frac{1}{1+\exp \left\{0,3 \times\left(-T_{\text {otima }}-10+T_{\text {mensal }}\right)\right\}}
\end{aligned}
$$

em que $\mathrm{T}_{1}$ é o fator que descreve os efeitos que surgem por causa do ar frio; $\mathrm{T}_{2}$ é um fator de redução da eficiência do uso da radiação decorrente do ar quente; $\mathrm{T}_{\text {otima }}$ é a média da temperatura do ar $\left({ }^{\circ} \mathrm{C}\right)$ durante o mês de máximo índice de área foliar ou NDVI; $\mathrm{T}_{\text {mensal }}$ é a média mensal da temperatura do ar $\left({ }^{\circ} \mathrm{C}\right)$.

Com a determinação de $\varepsilon_{f}$, obteve-se a biomassa vegetal acima do solo (Figura 4) por meio do modelo proposto por Monteith (1972) e utilizado por Bastiaanssen e Ali (2003):

$$
\text { Bio }=\varepsilon_{f} \times 0,864 \times \sum(A P A R(t))
$$

em que Bio é a biomassa vegetal no período t correspondente ao ciclo da cultura, ou seja, do plantio à colheita para a cana-planta ou do corte $n$ ao corte $n+1$ para a cana-soca. Na equação acima, utilizou-se o valor de 0,864 para a transformação de unidades e a obtenção da biomassa vegetal em kg ha ${ }^{-1}$ (Teixeira et al., 2012).

De posse da estimativa da biomassa vegetal foi possível obter a produtividade da cana-de-açúcar por meio da equação:

$$
Y_{C}=I_{C} \times \text { Bio }
$$

em que $\mathrm{Y}_{\mathrm{C}}$ é a produtividade da cultura $\left(\mathrm{kg} \mathrm{ha}^{-1}\right)$; $\mathrm{I}_{\mathrm{C}}$ é o índice de colheita. Para cana-de-açúcar, Bastiaanssen e Ali (2003) sugerem $\mathrm{I}_{\mathrm{C}}$ de 0,80 .

As estimativas de produtividade da cana-de-açúcar foram avaliadas por meio dos resultados estatísticos do erro absoluto médio (EAM), do erro padrão de estimativa (EPE), da raiz do erro quadrático médio (REQM) e do índice de concordância de Willmott (d) (Willmott, 1982).

\section{RESULTADOS E DISCUSSÃO}

A produção da cana-de-açúcar estimada utilizando o algoritmo SEBAL e imagem Landsat 5 TM variou de acordo com talhões em glebas com distintas épocas de plantios e colheitas (Figuras 5 a 9). Para a gleba A1 (Figura 5), a canade-açúcar foi plantada em 25/10/2006 e colhida em 31/8/2007 (talhões de 1 a 7) e 27/9/2007 (talhões de 8 a 10). A data de colheita antecipada deve-se a problemas com queimadas, entretanto esse fato não teve influência na cena de 7/8/2007 (ultima cena da série adquirida), pois a queimada aconteceu

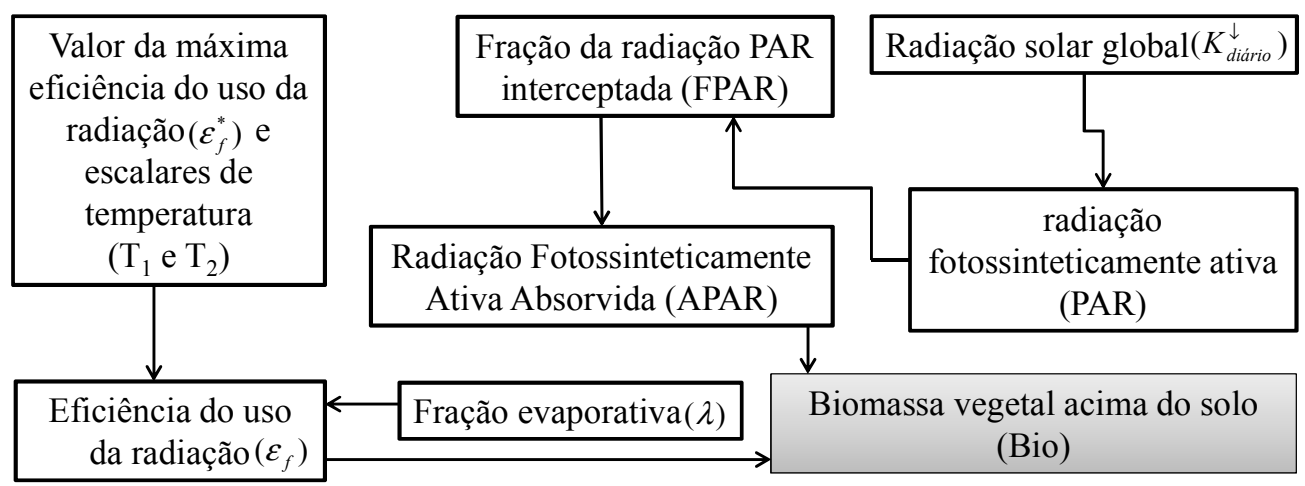

Figura 4 - Fluxograma da estimativa de biomassa vegetal acima do solo. 


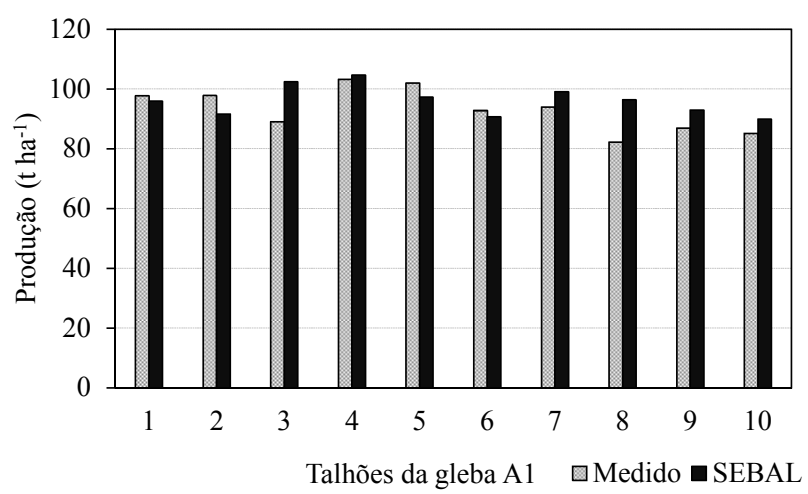

Figura 5 - Valores comparativos entre a produção de cana-de-açúcar medida e a estimada por meio da aplicação do SEBAL para os talhões da gleba A1.

poucos dias antes da colheita e, para que não ocorressem prejuízos, decidiu-se pelo corte.

A comparação estatística dos dados de produção observada com a produção estimada mostrou que a gleba A1 (Tabela 3, Figura 5) foi a que apresentou o segundo maior valor estatístico para o índice de concordância de $\operatorname{Willmott}(\mathrm{d}=0,70)$ e os menores valores para o erro absoluto médio $(\mathrm{EAM}=5,96$ $\left.\mathrm{t} \mathrm{ha}^{-1}\right)$, erro padrão de estimativa $\left(\mathrm{EPE}=7,70 \mathrm{tha}^{-1}\right)$ e raiz do erro quadrático médio $\left(\mathrm{REQM}=7,31 \mathrm{tha}^{-1}\right)$.

Vale ressaltar que, nos talhões 1, 4 e 6 da gleba A1, as estimativas foram bem próximas da produção observada, com diferença máxima de $2 \mathrm{t} \mathrm{ha}^{-1}$. Já os talhões 3 e 8 tiveram as maiores diferenças entre a produção estimada e a observada (até $\left.14 \mathrm{tha}^{-1}\right)$. Nos demais talhões, essa diferença variou entre $4 \mathrm{tha}^{-1}$ e 6 t ha $^{-1}$ (Figura 5). Uma possível justificativa para essa variação pode estar no fato de a produtividade ter sido influenciada por fatores como pragas, doenças e tratos culturais (Campos et al., 2010), que podem variar no tempo e no espaço, afetando, em diferentes níveis, a produção da cana-de-açúcar em cada talhão. A resolução espacial da imagem também é um fator que pode ter influenciado os resultados (Picoli et al., 2009), mas os erros de estimativa de produtividade da cana-de-açúcar para a gleba A1 estão dentro do esperado, quando comparados a outros métodos de estimativa encontrados na literatura.
Ao aplicar o método da zona agroecológica (método ZAE) na estimativa da produtividade da cana-de-açúcar para região do Triângulo Mineiro, Oliveira et al. (2012) obtiveram, para o primeiro ciclo da cana soca, valores de EAM e REQM da ordem de 6,7 tha- ${ }^{-1}$ e $8,4 \mathrm{t} \mathrm{ha}^{-1}$, respectivamente. Picoli et al. (2009) utilizaram índice de vegetação do sensor Modis para estimativa da produtividade da cana-de-açúcar na região oeste do Estado de São Paulo e obtiveram índice de concordância de Willmott de 0,87 e 0,62 para as safras 2004/2005 e 2005/2006, respectivamente. Além disso, observaram valores de EAM entre $12,1 \mathrm{t} \mathrm{ha}^{-1}$ (safra 2004/2005) e 15,3 $\mathrm{t} \mathrm{ha}^{-1}$ (safra 2005/2006) e valores de REQM de 20,9 $\mathrm{t} \mathrm{ha}^{-1}$ e 19,2 $\mathrm{t} \mathrm{ha}^{-1}$ para as safras 2004/2005 e 2005/2006, respectivamente.

Para a gleba A4 (Figura 6, Tabela 3), o valor de d foi de 0,66 e os valores de EAM, EPE e REQM foram de 18,96 t $\mathrm{ha}^{-1}, 24,21 \mathrm{tha}^{-1}$ e 22,65 tha ${ }^{-1}$, respectivamente. $\mathrm{O}$ talhão $1 \mathrm{com}$ plantio de cana-de-açúcar da variedade RB 86-7515 foi o que apresentou a menor diferença entre a produtividade estimada e a observada $\left(1,3 \mathrm{t} \mathrm{ha}^{-1}\right)$. Já as maiores diferenças foram registradas para os talhões 3,6 e 13, com valores da ordem de $34,8 \mathrm{tha}^{-1}$ (variedade RB 86-7515), 30,8 $\mathrm{tha}^{-1}$ (variedade SP 81-3250) e 35,6 tha ${ }^{-1}$ (variedades IAC 86-2480 e RB 92-8064), respectivamente.

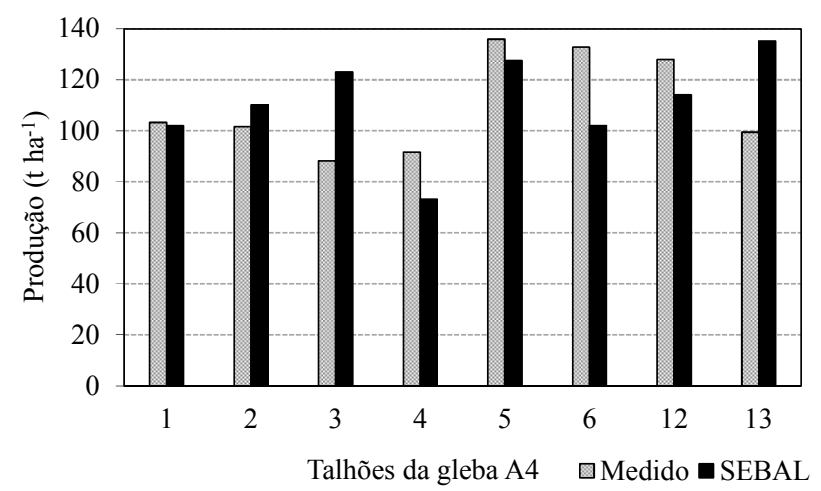

Figura 6 - Valores comparativos entre a produção da cana-de-açúcar medida e a estimada por meio da aplicação do SEBAL para os talhões da gleba A4.

Tabela 3 - Valores das estatísticas de concordância de Willmott (d), erro absoluto médio (EAM), erro padrão de estimativa (EPE) e raiz do erro quadrático médio (REQM) para avaliação da estimativa de produtividade da cana-de-açúcar nas glebas A1, A4, A5, E1, Pivô e considerando-se todas as glebas da fazenda Boa Fé utilizadas no estudo.

\begin{tabular}{ccccc}
\hline Glebas & d & EAM & EPE & REQM \\
\hline A1 & 0,70 & 5,96 & 7,70 & 7,31 \\
A4 & 0,66 & 18,96 & 24,21 & 22,65 \\
A5 & 0,79 & 11,28 & 16,14 & 13,97 \\
E1 & 0,47 & 15,74 & 19,32 & 17,88 \\
Pivô & 0,48 & 11,72 & 17,58 & 14,35 \\
\hline Todos & 0,64 & 12,55 & 16,34 & 16,08 \\
\hline
\end{tabular}


Apesar de os talhões 1 e 3 da gleba A4 serem idênticos em termos de variedade e período de plantio e colheita (Tabela 1), quando comparados observou-se grande diferença entre produtividade estimada e observada no talhão 3 . O formato dos talhões juntamente com a questão da resolução espacial da imagem podem ter influenciado esses resultados. Já para os demais talhões da gleba A4, além desses fatores, a variedade e a época de colheita também podem ter contribuído para essas maiores variações entre a produtividade estimada e a observada. Silva et al. (2008), ao estudar as variedades RB 72454 e IAC 86-2480, concluíram que a época de colheita interfere no perfilhamento e na produtividade da cultura.

Das glebas de plantio analisadas, a gleba A5 (Figura 7, Tabela 3) foi a que apresentou o maior índice de concordância de Willmott $(\mathrm{d}=0,79)$. Já os valores de EAM, EPE e REQM foram de 11,28 tha-1 $16,14 \mathrm{tha}^{-1}$ e 13,97 $\mathrm{t} \mathrm{ha}^{-1}$, respectivamente. Bastiaanssen e Ali (2003), ao utilizar o SEBAL juntamente com os modelos de eficiência do uso da radiação (Field et al., 1995) e acúmulo de biomassa (Monteith, 1972), com intuito de estimar a produção de algumas culturas na bacia de Indus, no Paquistão, obtiveram REQM de 13,484 t ha ${ }^{-1}$ para a cultura da cana-de-açúcar.

Na gleba A5, a maior diferença entre o valor de produção observada e estimada por meio do SEBAL foi registrada para o talhão $22\left(25,1 \mathrm{t} \mathrm{ha}^{-1}\right)$, já no talhão 24 , verificou-se a menor diferença (7,2 $\left.\mathrm{t} \mathrm{ha}^{-1}\right)$. A variedade RB 92-579 foi plantada no mesmo período em todos os talhões da gleba A5, porém, no talhão 24 , colheu-se a cana-de-açúcar aproximadamente um mês após a colheita dos demais talhões (Tabela 1).

Já na gleba E1 (Figura 8), com sete talhões de plantios da cana-de-açúcar, a menor e a maior diferença entre produção estimada e observada foram de $7,4 \mathrm{t} \mathrm{ha}^{-1}$ (talhão 4) e 31,5 $\mathrm{t} \mathrm{ha}^{-1}$ (talhão 5), respectivamente. Vale ressaltar que os talhões que registraram as maiores diferenças são aqueles de menor área, o que pode ter contribuído para que os valores de EAM, EPE e

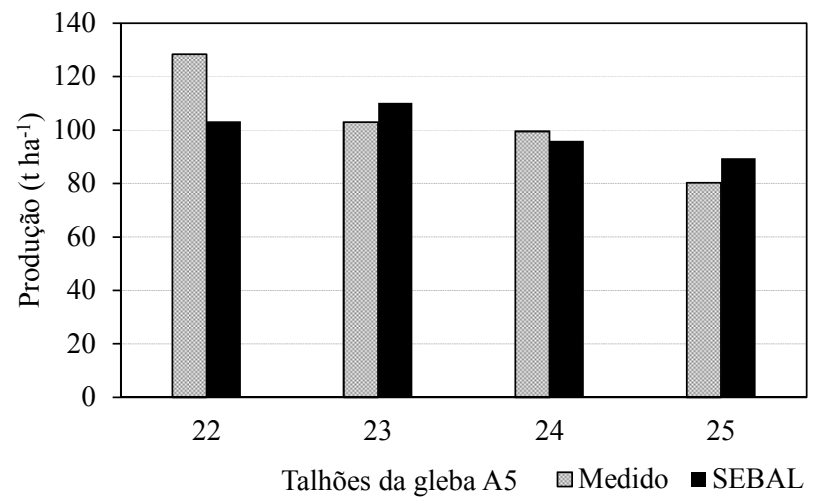

Figura 7 - Valores comparativos entre a produção da cana-de-açúcar medida e a estimada por meio da aplicação do SEBAL para os talhões da gleba A5.
REQM fossem apenas menores que os resultados encontrados para a gleba A4. Mas, quando se compara a produtividade da gleba A4, com a da gleba E1, nota-se que boa parte dos talhões da gleba A4 foram mais produtivos e, além disso, registraram maior variabilidade de produção entre talhões, provavelmente em decorrência do maior número de variedades cultivadas.

Na gleba Pivô, as estimativas de produção feitas por meio do SEBAL (Figura 9) apresentaram erros de 3,9 $\mathrm{tha}^{-1}, 8,1 \mathrm{tha}^{-1} \mathrm{e}$ $23,2 \mathrm{t} \mathrm{ha}^{-1}$, respectivamente, para os talhões 1,2 e 3. Das glebas avaliadas, as glebas E1 e pivô foram as que apresentaram os menores valores de concordância de Willmott $(d=0,48$, Tabela 3). No entanto, para a gleba pivô os valores de EAM, EPE e REQM foram próximos aos encontrados para a gleba A5, que teve o maior valor de d. Destaca-se também que os três talhões da gleba pivô são cultivados com a mesma variedade de canade-açúcar (RB 867515) e que, em relação às demais glebas, os talhões da gleba pivô são os que têm a maior área de cultivo e as maiores produtividades. Uma das justificativas pode estar na utilização de irrigação suplementar nos primeiros meses de desenvolvimento da cultura.

De forma geral, as análises de concordância de Willmott (d) apresentaram variação na exatidão das estimativas de acordo com cada gleba do estudo, e variaram de 0,47 na gleba E1 a 0,79 na gleba A5. Quanto aos valores de EAM, EPE e REQM, verificou-se que, nas glebas A1 e A4, foram registrados os menores e os maiores erros, respectivamente. Em uma análise geral, na qual os talhões não foram separados por glebas, a concordância de Willmott foi de 0,64.

\section{CONCLUSÕES}

A aplicação de técnicas de sensoriamento remoto juntamente com o algoritmo SEBAL apresentou variação de desempenho nas estimativas de produtividade da cana-de-açúcar em cada gleba, provavelmente em decorrência de influências

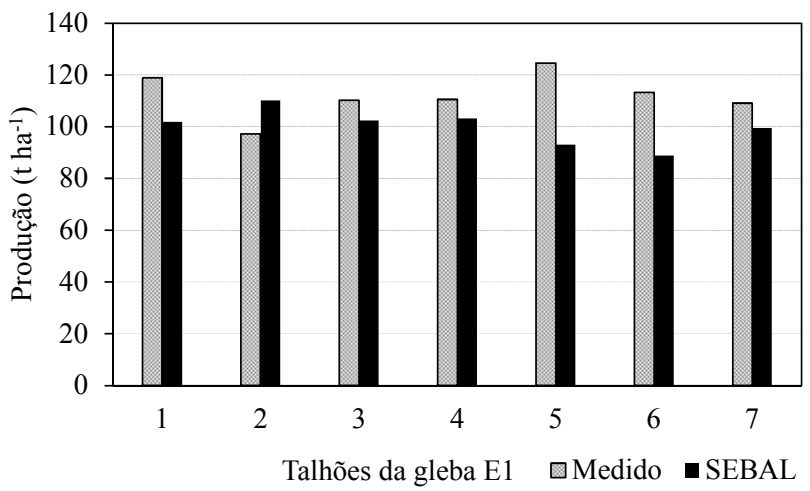

Figura 8 - Valores comparativos entre a produção da cana-de-açúcar medida e a estimada por meio da aplicação do SEBAL para os talhões da gleba E1. 


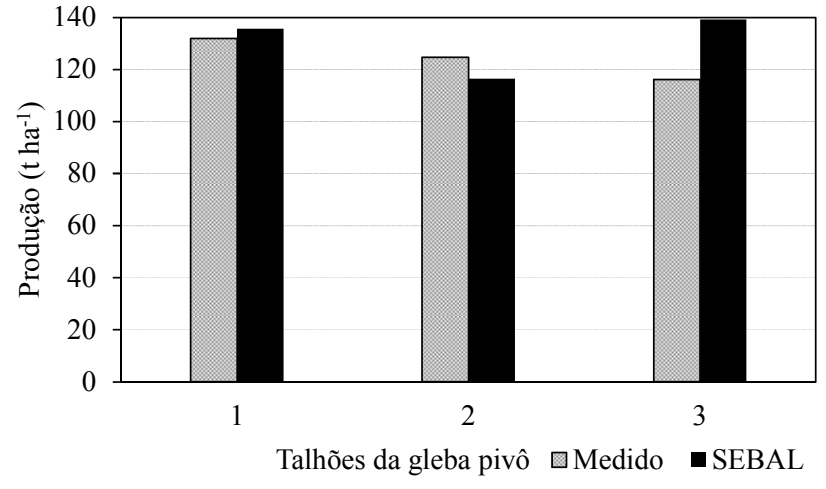

Figura 9 - Valores comparativos entre a produção da cana-de-açúcar medida e a estimada por meio da aplicação do SEBAL para os talhões da gleba pivô.

da dimensão dos talhões e da resolução espacial da imagem, de variedades e épocas de plantio e colheita da cultura. Porém, de forma geral, os resultados obtidos mostram que a metodologia tem potencial para ser aplicada em áreas extensas e com limitada disponibilidade de dados meteorológicos.

\section{AGRADECIMENTOS}

À FAPEMIG, pelo financiamento do projeto de pesquisa, e à CAPES e ao CNPq, pelas bolsas de doutorado concedidas nos dois anos iniciais e finais da pesquisa, respectivamente. Ao Grupo Ma Shou Tao, pela atenção e pela disponibilização de alguns dados da fazenda Boa Fé.

\section{REFERÊNCIAS BIBLIOGRÁFICAS}

ALLEN, R.G.; TASUMI, M.; TREZZA, R.; WATERS, R.; BASTIAANSSEN, W. SEBAL: surface energy balance algorithms for land. Idaho implementation, advanced training and user's manual. Version 1.0. Idaho: University of Idaho, 2002. 98p.

ANDRADE, R.G.; SEDIYAMA, G.C.; PAZ, A.R.; LIMA, E.P.; FACCO, A.G. Geotecnologias aplicadas à avaliação de parâmetros biofísicos do Pantanal. Pesquisa Agropecuária Brasileira, v.47, n.9, p.1227-1234, 2012.

BASTIAANSSEN, W.G.M.; ALI, S. A new crop yield forecasting model based on satellite measurements applied across the Indus Basin, Pakistan. Agriculture Ecosystems \& Environment, v.94, p.321-340, 2003.

BASTIAANSSEN, W.G.M.; MENENTI, M.; FEDDES, R.A.; HOLTSLAG, A.A.M. A remote sensing surface energy balance algorithm for land (SEBAL) 1. Formulation. Journal of Hydrology, v.212-213, p.198-212, 1998a.

BASTIAANSSEN, W.G.M.; PELGRUM, H.; WANG, J.; MA, Y.; MORENO, J.F.; ROERINK, G.J.; VAN DER WAL, T.
A remote sensing surface energy balance algorithm for land (SEBAL): 2. Validation. Journal of Hydrology, v.212-213, p.213-229, 1998b.

BASTIAANSSEN, W.G.M. SEBAL - based sensible and latent heat fluxes in the irrigated Gediz Basin, Turkey. Journal of Hydrology, v.229, p.87-100, 2000.

CAMPOS, L.H.F.; CARVALHO, S.J.P.; CHRISTOFFOLETI, P.J.; FORTES, C.; SILVA, J.S. Sistemas de manejo da palhada influenciam acúmulo de biomassa e produtividade da cana-de-açúcar (var. RB855453). Acta Scientiarum. Agronomy, v.32, n.2, p.345-350, 2010.

CARVALHO, S.P.; MARIN, J.O.B. Agricultura familiar e agroindústria canavieira: impasses sociais. Revista de Economia e Sociologia Rural, v.49, n.3, p.681-707, 2011.

DAUGHTRY, C.S.T.; GOWARD, K.P.; PRINCE, S.D.; KUSTAS, W.P. Spectral estimates of absorbed radiation and phytomass production in corn and soybean canopies. Remote Sensing of Environment, v.39, p.141-152, 1992.

FIELD, C.B., RANDERSON, J.R., MALMSTRÖM, C.M. Global net primary production: combining ecology and remote sensing. Remote Sensing of Environment, v. 51, p. 74-88, 1995.

GUIMARÃES, L.T.; TURETTA, A.P.D.; COUTINHO, H.L.C. Uma proposta para avaliar a sustentabilidade da expansão do cultivo da cana-de-açúcar no estado do Mato Grosso do Sul. Sociedade \& Natureza, v.22, n.2, 2010.

MELLO, C.O.; ESPERANCINI, M.S.T. Análise econômica da eficiência da produção de cana-de-açúcar de fornecedores do estado do Paraná. Revista Energia na Agricultura, v.27, n.3, p. 48-60, 2012.

MENEZES, S.J.M.C.; SEDIYAMA, G.C.; SOARES, V.P.; GLERIANI, J.M.; ANDRADE, R.G. Estimativa dos componentes do Balanço de Energia e da Evapotranspiração em Plantios de Eucalipto utilizando o algoritmo SEBAL e imagem Landsat 5-TM. Revista Árvore, v.35, p.649-657, 2011.

MONTEITH, J.L. Solar radiation and productivity in tropical ecosystems. Journal of Applied Ecology, v.9, p.747-766, 1972.

OLIVEIRA, R.A.; SANTOS, R.S.; RIBEIRO, A.; ZOLNIER, S.; BARBOSA, M.H.P. Estimativa da produtividade da cana-de-açúcar para as principais regiões produtoras de Minas Gerais usando-se o método ZAE. Revista Brasileira de Engenharia Agrícola e Ambiental, v.16, n.5, p.549557, 2012.

PICOLI, M.C.A.; RUDORFF, B.F.T.; RIZZI, R.; GIAROLLA, A. Índice de vegetação do sensor MODIS na estimativa da produtividade agrícola da cana-de-açúcar. Bragantia, v.68, n.3, p.789-795, 2009. 
SAMARASINGHE, G.B. Growth and yields of Sri Lanka's major crops interpreted from public domain satellites. Agricultural Water Management, v.58, p.145-157, 2003.

SILVA, B.B.; BEZERRA, M.V.C. Determinação dos fluxos de calor sensível e latente na superfície utilizando imagens TM - Landsat 5. Revista Brasileira de Agrometeorologia, v.14, n.2, p.174-186, 2006.

SILVA, B.B.; BRAGA, A.C.; BRAGA, C.C.; OLIVEIRA, L.M.M.; GALVÍNCIO, J.D.; MONTENEGRO, S.M.G.L. Evapotranspiração e estimativa da água consumida em perímetro irrigado do Semiárido brasileiro por sensoriamento remoto. Pesquisa Agropecuária Brasileira, v.47, p.12181226, 2012.

SILVA, M.A.; JERONIMO, E.M.; LÚCIO, A.D. Perfilhamento e produtividade de cana-de-açúcar com diferentes alturas de corte e épocas de colheita. Pesquisa Agropecuária Brasileira, v.43, n.8, p.979-986, 2008.
TEIXEIRA, A. H. C.; SHERER-WARREN, M.; HERNANDEZ, F. B. T.; LOPES, H. L. Water productivity assessment by using MODIS images and agrometeorological data in the Petrolina municipality, Brazil. In: SPIE remote sensing symposium, 2012, Edimburgo. Proceedings of SPIE, 2012. v. 8531.p. 85310G-1-85310G-10.

VICENTE, L.E.; GOMES, D.; VICTORIA, D.C.; GARÇON, E.A.M.; BOLFE, E.L.; ANDRADE, R.G.; SILVA, G.B.S. Séries temporais de NDVI do sensor SPOT Vegetation e algoritmo SAM aplicados ao mapeamento de cana-deaçúcar. Pesquisa Agropecuária Brasileira, v.47, n.9, p.1337-1345, 2012.

WILLMOTT, C.J. Some comments on the evaluation of model performance. Bulletin of the American Meteorological Society, v.63, n.11, p.1309-1313, 1982. 